High Pressure Burn Rate Measurements on an Ammonium Perchlorate Propellant

E. A. Glascoe, N. Tan

April 23, 2010 
This document was prepared as an account of work sponsored by an agency of the United States government. Neither the United States government nor Lawrence Livermore National Security, LLC, nor any of their employees makes any warranty, expressed or implied, or assumes any legal liability or responsibility for the accuracy, completeness, or usefulness of any information, apparatus, product, or process disclosed, or represents that its use would not infringe privately owned rights. Reference herein to any specific commercial product, process, or service by trade name, trademark, manufacturer, or otherwise does not necessarily constitute or imply its endorsement, recommendation, or favoring by the United States government or Lawrence Livermore National Security, LLC. The views and opinions of authors expressed herein do not necessarily state or reflect those of the United States government or Lawrence Livermore National Security, LLC, and shall not be used for advertising or product endorsement purposes.

This work performed under the auspices of the U.S. Department of Energy by Lawrence Livermore National Laboratory under Contract DE-AC52-07NA27344. 


\title{
High Pressure Burn Rate Measurements on an Ammonium Perchlorate Propellant
}

\author{
Final Report \\ 4/20/2010 \\ Dr. Elizabeth Glascoe and Noel Tan \\ Lawrence Livermore National Laboratory \\ Energetic Materials Center \\ 7000 East Ave., Livermore, CA 94550
}

\section{Introduction}

High pressure deflagration rate measurements of a unique ammonium perchlorate (AP) based propellant are required to design the base burn motor for a Raytheon weapon system. The results of these deflagration rate measurements will be key in assessing safety and performance of the system. In particular, the system may experience transient pressures on the order of 100's of MPa (10's kPSI). Previous studies on similar AP based materials demonstrate that low pressure (e.g. P $<10 \mathrm{MPa}$ or $1500 \mathrm{PSI}$ ) burn rates can be quite different than the elevated pressure deflagration rate measurements (see Ref ${ }^{1}$ and HPP results discussed herein), hence elevated pressure measurements are necessary in order understand the deflagration behavior under relevant conditions.

Previous work on explosives have shown that at 100's of MPa some explosives will transition from a laminar burn mechanism to a convective burn mechanism in a process termed deconsolidative burning. The resulting burn rates that are orders-of-magnitude faster than the laminar burn rates. ${ }^{2}$ Materials that transition to the deconsolidativeconvective burn mechanism at elevated pressures have been shown to be considerably more violent in confined heating experiments (i.e. cook-off scenarios). ${ }^{3}$ The mechanisms of propellant and explosive deflagration are extremely complex and include both chemical, and mechanical processes, hence predicting the behavior and rate of a novel material or formulation is difficult if not impossible.

In this work, the AP/HTPB based material, TAL-1503 (B-2049), was burned in a constant volume apparatus in argon up to $300 \mathrm{MPa}$ (ca. $44 \mathrm{kPSI}$ ). The burn rate and pressure were measured in-situ and used to calculate a pressure dependent burn rate. In general, the material appears to burn in a laminar fashion at these elevated pressures. The experiment was reproduced multiple times and the burn rate law using the best data is

$$
B=(0.6 \pm 0.1) \times P^{(1.05 \pm 0.02)}
$$

where $B$ is the burn rate in $\mathrm{mm} / \mathrm{s}$ and $P$ is the pressure in units of MPa. Details of the experimental method, results and data analysis are discussed herein and briefly compared to other AP based materials that have been measured in this apparatus.

\section{Method}

\section{Instrument}

The LLNL High Pressure Strand Burner, shown schematically in Figure F1, combines the features of a traditional closed-bomb burner with those of a traditional strand burner. It contains a deflagrating sample in a small volume, high-pressure chamber. Simultaneous temporal pressure and burn front time-of-arrival measurements yield the laminar deflagration rate for a range of pressures and provide insight into deflagration uniformity in one experiment. Pressure is measured using a pressure transducer and a load cell, and burn front arrival is detected by the burning-through of thin silver wires embedded in the sample. High speed digital scopes capture the data for subsequent analysis.

The strand burner has an internal volume of approximately $75 \mathrm{~cm}^{3}\left(4.6 \mathrm{in}^{3}\right)$ and is designed to reach pressures of $1 \mathrm{GPa}(150,000 \mathrm{psi})$. The pressure vessel body is built from two concentric shells with interference between them to put the inner shell in compression. The inner liner and the top closures are fabricated from Hasteloy and Ultimate, respectively; both materials are corrosive resistant steels designed to prevent corrosive stress cracking by chlorine radicals. The strength of these steels ultimately limits the operational pressure range of the apparatus to $\mathrm{P} \leq 300 \mathrm{MPa}$; higher pressures may be feasible but the equipment has never been tested above $300 \mathrm{MPa}$. The top plug is equipped with gas inlet and outlet ports and a pressure transducer, while the bottom plug holds a prewired base and high pressure feed-throughs for the burn wires, igniter wires, and thermocouples. The pressure transducer is a Kistler model 6213B. The load cell is Omega model LCTB-150K. The commercial sensor was calibrated to NIST standards by the manufacturer, and the load cell was calibrated against the Kistler transducer. 


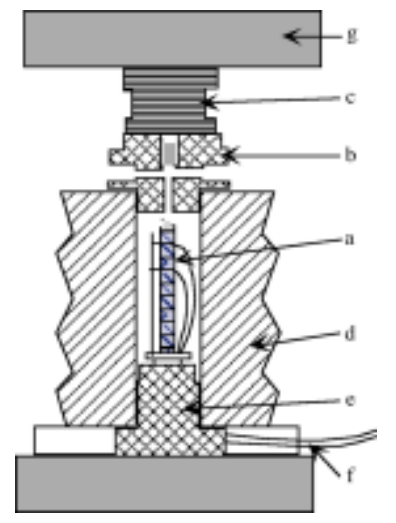

Figure F1. Strand burner schematic composed of a) nine segment burn sample with burn wires evenly spaced between segments (only two wires shown for clarity) and igniter on top, b) top plug with inlet and outlet ports and pressure transducer in center, c) load cell, d) pressure vessel, e) bottom plug with wire feed-throughs, f) signal wires to electronics, g) load frame (top and bottom).

\section{Sample}

Tal-1503 B-2049 is an AP/HTPB formulation with $74 \mathrm{wt} \%$ AP, $16.9 \mathrm{wt} \%$ HTPB and $9.1 \mathrm{wt} \%$ other constituents. Small pellets ( $1 / 4$ inch diameter by $1 / 4$ inch height) were supplied by Nammo Talley; Figure F2 (left) shows the pellets as supplied. The pellets all have smooth top and bottom surfaces which are important for creating intimate contact between pellets when they are stacked; the rough circumference and variable radius are less critical and most likely do not interfere with the burn measurements although they may be one source of error in the experiments (see discussion section). Each pellet is filed on one end (see Figure F2, right) in order to create a groove for the burn wire to rest in. The pellets are then assembled in a column consisting of 9 pellets, see Figure F3, with one burn wire between each pellet. Burn wires consist of seventy-five-micron ( 3 mil) diameter silver wire that is coated with a thin polymeric material to prevent wire cross-talk. After assembly, the cylindrical surface of the sample is coated with Halthane 88-2 (an organic polymeric material) to inhibit burning on the outer surface, see Figure F4. This limits the flame front to the cross-sectional surface of the cylinder. An igniter train consisting of an igniter wire, approximately $110 \mathrm{mg}$ of $\mathrm{BKNO}_{3}$, and a thin, $35 \mathrm{mg}$ pressed HNS pellet ignites the burn sample on the top end of the cylinder.
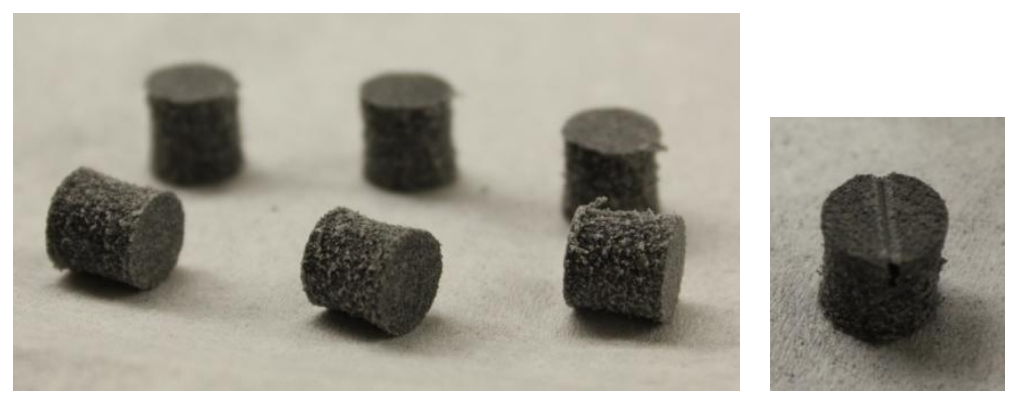
Figure F2. Representative propellant pellets as supplied by Nammo Talley (left) and after filing (right). A single groove was hand-filed in each pellet to make space for a single burn wire. Pellets are assembled into a strand as shown in Figures F3 and F4.

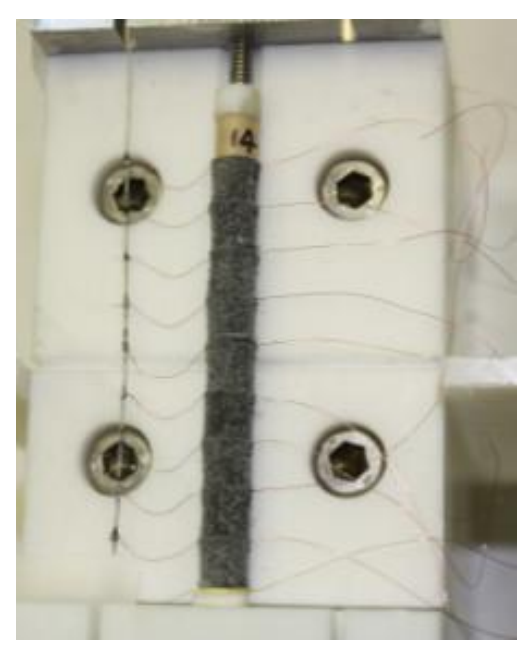

Figure F3. A propellant strand prior to applying Halthane 88-2 (i.e. rubber encapsulation). Burn wires are embedded between pellets in hand-filed slots (see Figure F2). $\mathrm{BKNO}_{3}$ and $\mathrm{HNS}$ igniter are packed into the cardboard tube on top.

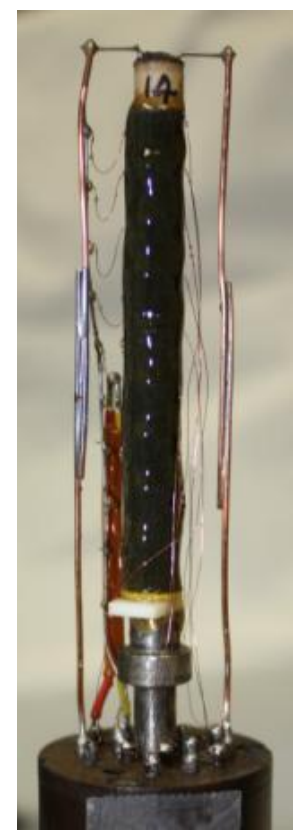

Figure F4. Propellant strand prior to burn. Strand is encapsulated in Halthane 88-2 in order to prevent flame flashing down the side of the strand and to hold assembly together. Burn wires are soldered to a base that is connected to an oscilloscope. A wire is embedded in the cardboard tube containing the $\mathrm{BKNO}_{3}$ and $\mathrm{HNS}$ pellet and is used to ignite the materials.

\section{Experiment Measurement}


To conduct a measurement, the sample is mounted into a pre-wired base that carries the signal wires through high pressure feed-throughs in the bottom plug of the pressure vessel, see Figure F4. The sample and bottom plug are then inserted into the pressure vessel. The system is pressurized to the desired starting pressure with argon (up to $115 \mathrm{MPa}$ or 16,500 psi in these experiments) and remotely sealed by immersing the inlet and outlet argon gas lines in liquid nitrogen. The argon freezes and acts as a remotely actuated pressure isolation valve with no moving parts or seals to maintain. Once the desired pressure is reached, sample deflagration is initiated, and temporal pressure and burn front time-of-arrival data are recorded. Following the experiment, the pressure is released by remotely removing the outlet argon gas line from the liquid nitrogen.

As the burn progresses down the strand, each burn wire burns and breaks creating a timestamp for the burn progress. The burn wire electronics provide a well defined signal, with measured rise times less than 40 microseconds. The wires burn through reproducibly, with wires mounted at the same location in the sample showing a standard deviation of 1-2 milliseconds. The wires do take several milliseconds to burn through; however, this time is essentially independent of initial pressure and temperature conditions and therefore does not affect the deflagration rate calculation by differences in time-of-arrival. The burn wire at the bottom of the stack does not burn through unless enough energetic material is placed below it to provide several milliseconds of burning once the flame front has passed; in this case LX-04 (85\% HMX, 15\% Viton-A) was used. Burn wires are recorded in a way which allows for unambiguous assignment of each signal to a particular wire. This is necessary, as wires occasionally report out of sequence if broken by debris in the bomb chamber.

\section{Calculations}

To calculate the deflagration rate as a function of pressure, the length and time-of-arrival for each pair of pellets is used, and the corresponding average pressure for this segment of the sample is calculated. The pressure dependent deflagration rate is fit to the Vieille equation:

$$
B=a P^{n}
$$

where $B$ is the burn rate $(\mathrm{mm} / \mathrm{s}), a$ is the burn rate coefficient ( $\mathrm{mm} / \mathrm{s} \cdot \mathrm{MPa}), P$ is the pressure (MPa), and $n$ is the pressure exponent (dimensionless).

\section{Results}

\section{Small Scale Safety Testing}

Prior to assembling strands and performing deflagration rate experiments, TAL-1503 was subjected to a battery of standard small scale safety tests in order to establish safe methods of working with the material. At the LLNL High Explosives Application Facility there are six standard small scale safety tests: impact (i.e. drop hammer), friction, spark, thermal decomposition (i.e. DSC), thermal stability (i.e. vacuum stability), and chemical compatibility. Results of these tests are listed in Table T1. The drop hammer test results are for a 50\% go/no-go result, i.e. at the height reported, five out of ten tests resulted in a reaction of the material. The friction test reports the weight necessary to get one reaction out of ten attempts. The spark test is performed by measuring the number of reactions (out of 10) using a 1.0 Joule electrostatic discharge. Two different differential scanning calorimetry (DSC) measurements are made in order to establish the onset of thermal decomposition. Both tests are performed at 10 ${ }^{\circ} \mathrm{C} / \mathrm{min}$ and the pan type is varied (hermetic closed vs. hermetic pinhole) in order to probe the possible effects of air and pressure in the reaction. The thermal stability test is a modified vacuum stability test in which a sample is heated under vacuum for 22 hours at $120^{\circ} \mathrm{C}$ and the volume of gas produced is measured. The results of the thermal stability test are reported in $\mathrm{cm}^{3}$ of product gas per gram of propellant material. Finally, chemical compatibility is measured using the thermal stability technique but the materials of interest are mixed and the mixture gas volume is compared with the constituent gas volumes produced. Both the thermal stability and the chemical compatibility test must have gas product volumes that are less than a facility designated level. In this case, TAL-1503 passed the thermal stability test and passed the compatibility test with the materials listed in Table 1.

Table 1. Results of LLNL small scale safety tests on TAL-1503.

\begin{tabular}{|c|c|c|c|c|c|}
\hline $\begin{array}{c}\text { Impact } \\
\text { (drop hammer) }\end{array}$ & Friction & $\begin{array}{c}\text { Spark } \\
(\mathrm{ESD})\end{array}$ & $\begin{array}{c}\text { Thermal Decomp. Onset } \\
\left(\mathrm{DSC} \text { at } 10^{\circ} \mathrm{C} / \mathrm{min}\right)\end{array}$ & $\begin{array}{c}\text { Thermal } \\
\text { Stability }\end{array}$ & Compatible Chemicals \\
\hline
\end{tabular}




\begin{tabular}{|c|c|c|c|c|c|}
\hline $101 \mathrm{~cm}$ & $\begin{array}{c}1 / 10 @ \\
16.8 \mathrm{~kg}\end{array}$ & $\begin{array}{c}0 / 10 @ \\
1.0 \text { Joule }\end{array}$ & $\begin{array}{c}\text { Approx. } 210^{\circ} \mathrm{C} \\
\text { (hermetic closed pan) } \\
\text { Approx. 225 } \mathrm{C} \\
\text { (hermetic pinhole pan) }\end{array}$ & $\begin{array}{c}0.11 \mathrm{~cm}^{3} / \mathrm{g} \\
\text { (after 22hrs } \\
\text { at } 120^{\circ} \mathrm{C} \text { ) }\end{array}$ & $\begin{array}{c}\text { Halthane 88-2 } \\
\text { LX-04 }\end{array}$ \\
HNS/silver wire/Teflon \\
\hline
\end{tabular}

\section{Raw Data}

In this study, four strands were burned at different initial pressures. The first strand (burn number 10018) was burned at a relatively low pressure of 20-45 MPa (ca. 3-6.5 kPSI) in order establish the nature of the material burn without damaging the equipment and to provide a comparison with deflagration rate measurements performed in other, lower pressure systems. The remaining three strands were burned at elevated pressure ranging from 80 $300 \mathrm{MPa}$; this was the pressure region of interest and the maximum pressure safely attainable in the system. It is in this higher pressure region where materials can often transition to the rapid and erratic convective burn mechanism; hence multiple experiments were performed to ensure reproducibility.

Figure F5 shows the pressure and flame front time-of-arrival data for one of the strands burned in this study (specifically burn number 10018). These results represent show a well behaved burn: the wires are burned in order and the pressure rise stops after the last burn wire. Burn number 10019 and 10020 both appear more erratic (see Figures F6 and F7). In particular, 10019 burned two wires out of order, wire 3 burned at the same time as wire 5 and wire 7 and 8 burned at the same time. The data was analyzed with the report times for wires 3 and 8 removed; analysis of the data with wires 3 and 7 removed was also attempted and the results were generally the same regardless of whether wire 7 or 8 was left out.

Neither burn 10019 nor burn 10020 are considered "bad" burns, the most important criteria have been satisfied: the wires burn in order (for the most part) and burn wire report times are generally well correlated with the pressure rise. The burn wire data should cover the time span of the pressure signal. Any significant deviation from this indicates anomalous behavior. For example, the report of all burn wires before the pressure reaches a maximum indicates that the deflagration front passes rapidly down the sample and leaves still-reacting material behind. This behavior is indicative of flame spread through the sample or of propagation of the flame down the side of the sample; however, the rubber coating should inhibit the latter. Instead burn 10019 and 10020 are just a bit erratic, the reason for these this erratic burns is unknown, however, the fact that burn number 10021(see Figure F8) is so smooth and consistent indicates that the erratic burns may be due to small random variables. For example, the Halthane coating on strand number 10019 and 10020 could be a little thin in places allowing for a small amount of local flame flashing over a short distance. Alternatively, a small amount of glue may have seeped in between the pellets causing some small inhibition of the burn (this may explain why burn wires 3 and 7/8 burned out of order in 10019). As was shown in Figure F2, the cylindrical surfaces of the pellets in all the strands are a little rough and have variable radius, hence there may be some small local patches that were not glued as smoothly or the burn transition from one pellet to the next may be a little erratic due to mismatch of the pellet radii at the junction (there may be some glue on the larger pellet surface that hinders the burn a little).



Figure F5. Pressure and burn wires data for burn number 10018 . 


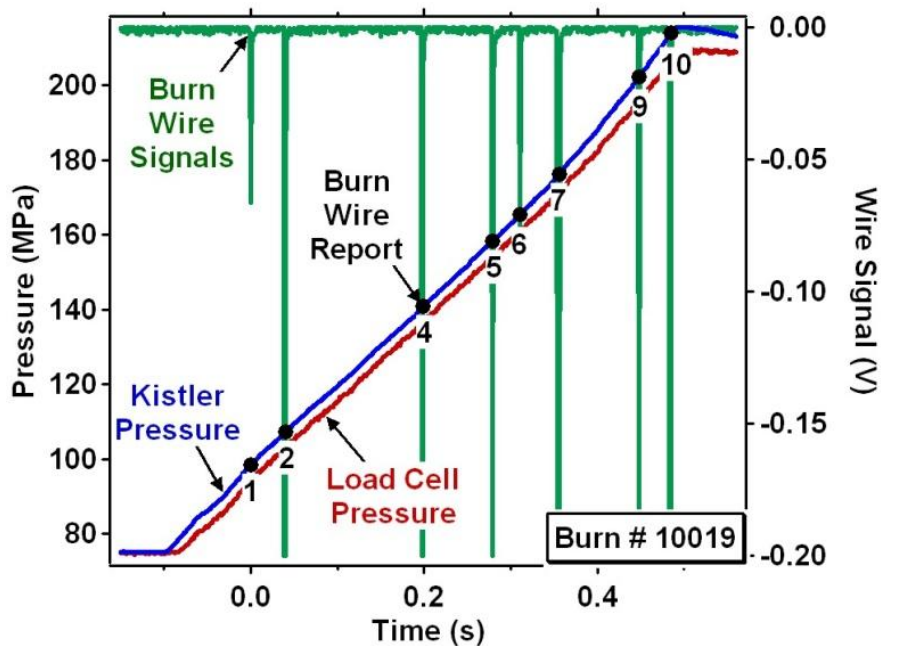

Figure F6. Pressure and burn wires data for burn number 10019. Data from wires 3 and 8 were removed for analysis.

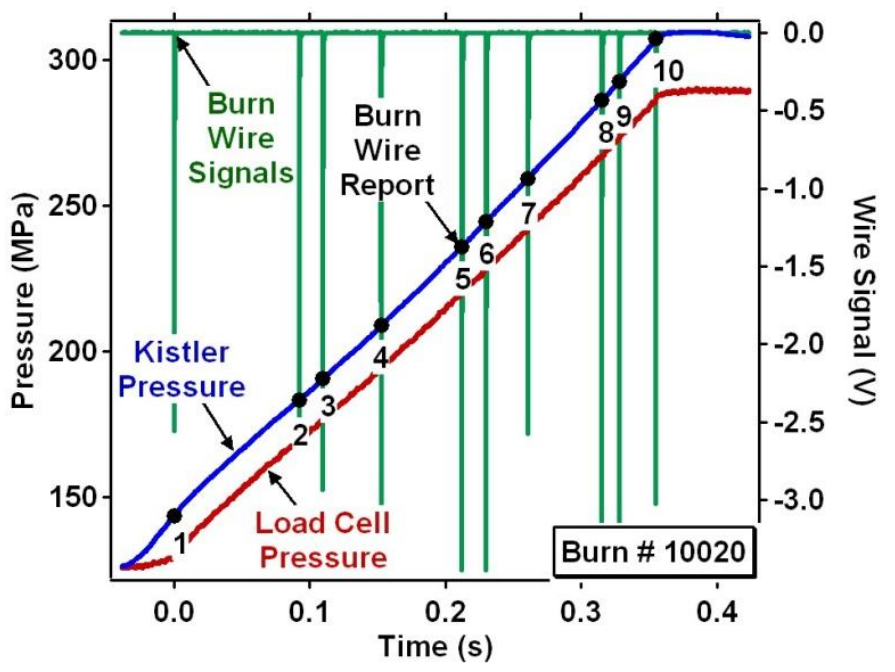

Figure F7. Pressure and burn wires data for burn number 10020. 




Figure F8. Pressure and burn wires data for burn number 10021.

The pressure dependent burn rate was calculated by taking the time between burn wires and the length of each pellet (measured prior to assembly) and calculating a burn rate for each pellet and using the average/median pressure experienced during the burning of that pellet. An averaging method in which adjacent wires (e.g. 1 and 3) and the associated distance of two pellets was used to acquire a smoother set of data. The pressure and corresponding burn rate for each strand is listed in Table 1. The pressure dependent burn rate is plotted in Figure F9; it is clear that burn number 10019 and 10020 are considerably more erratic than 10018 and 10021. The criteria for a well-behaved burn are discussed above. In general, there are a variety of possible random errors that can occur during a burn, however, the likelihood of significant random errors producing a well-behaved burn are small. Therefore, the orderly nature of the results for burns 10018 and 10021 indicate that these data are representative of experiments that are free of significant random errors. The erratic nature of 10019 and 10020 are most likely due to small random variables, however, it is encouraging to see that they burn, in general, with a similar burn rate to that observed in 10021.

\begin{tabular}{|c|c|c|c|c|c|c|c|}
\hline $\begin{array}{c}\text { Pressure } \\
(\mathrm{MPa}) \\
\# 10018\end{array}$ & $\begin{array}{c}\text { Burn Rt. } \\
(\mathrm{mm} / \mathrm{s})\end{array}$ & $\begin{array}{c}\text { Pressure } \\
(\mathrm{MPa})\end{array}$ & $\begin{array}{c}\text { Burn Rt. } \\
(\mathrm{mm} / \mathrm{s})\end{array}$ & $\begin{array}{c}\text { Pressure } \\
(\mathrm{MPa}) \\
\# 10018\end{array}$ & $\begin{array}{c}\text { Burn Rt. } \\
(\mathrm{mm} / \mathrm{s})\end{array}$ & $\begin{array}{c}\text { Pressure } \\
(\mathrm{MPa}) \\
\# 10019\end{array}$ & $\begin{array}{c}\text { Burn Rt. } \\
(\mathrm{mm} / \mathrm{s}) \\
\# 10019\end{array}$ \\
\hline 22.0139 & 15.8652 & 119.701 & 99.2517 & 167.262 & 120.124 & 162.957 & 109.708 \\
24.9722 & 17.9814 & 132.857 & 82.5861 & 196.096 & 217.162 & 185.515 & 135.8 \\
27.2118 & 15.7533 & 153.222 & 116.737 & 213.377 & 127.94 & 206.28 & 157.342 \\
29.5972 & 17.467 & 167.399 & 170.145 & 226.783 & 168.787 & 222.254 & 174.504 \\
32.191 & 22.7497 & 183.909 & 143.505 & 247.679 & 269.545 & 240.296 & 194.655 \\
34.9722 & 25.1476 & 195.191 & 153.73 & 265.346 & 155.918 & 256.046 & 189.907 \\
37.9722 & 25.6997 & & & 275.997 & 196.808 & 271.963 & 203.36 \\
41.3577 & 28.8875 & & & 296.585 & 332.504 & 288.015 & 217.13 \\
\hline
\end{tabular}

Table 1. Burn rate results for TAL-1503 from each individual strand/experiment (10018, 10019, 10020, and 10021 are experiment numbers)

A burn law fit was determined using Vieille's law (see equation 1). Two different burn law fits were attempted, the first using all the data, the second using just the best two burns (i.e. 10018 and 10021). The errors reported in the fits in Figure F9 correspond to one sigma and the goodness of the fit to the data. Variability associated with independent variables such as the pellet length, the groove distance, the pressure response time, etc, are not included in this error; in general these variables are sufficiently small that they play a minor role in the overall burn rate. The results in Figure F9 indicate that fitting all the data versus just the "best" data does not change the parameters outside of the error. In general, the results of the "best data" fit are recommended for use in any further analysis. 


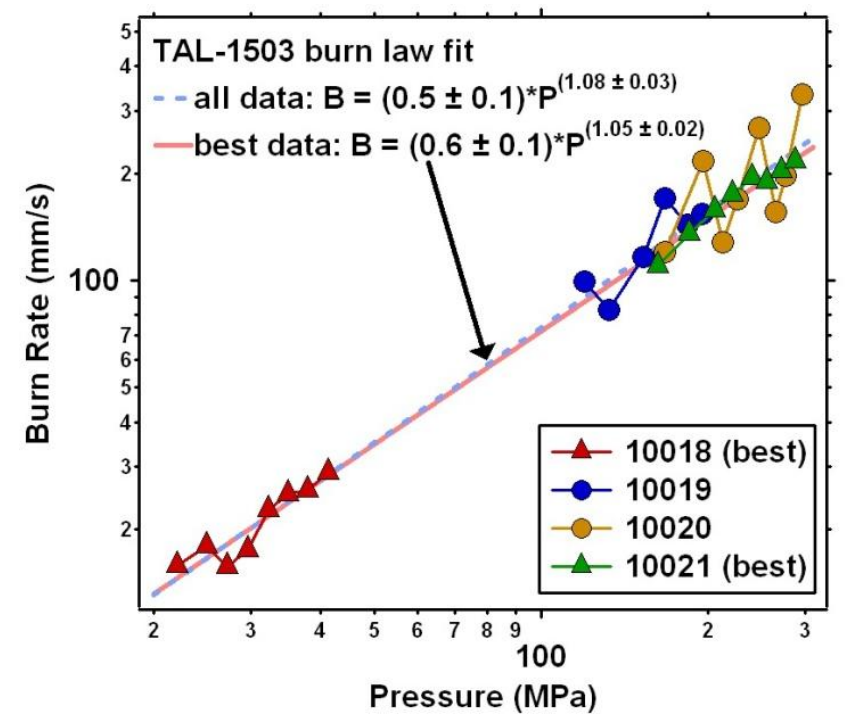

Figure F9. Results of TAL-1503 LLNL high pressure strand burner experiments. Each color represents a different strand and therefore an individual experiment. The two experiments depicted with the triangles (\#10018 and 10021) appear to be the most stable and least erratic and a burn rate fit to just these two experiments is reported. A burn rate fit to all the data is also included.

\section{Discussion}

Comparison of TAL-1503 with other AP based propellants and other energetic materials are interesting. Previously at LLNL, we've studied AP based propellants with micrometer sized aluminum. Figure F10 shows the TAL-1503 data plotted with HPP and PS-4, both are AP/Al based propellants; all three materials have similar AP particle size distributions and amounts. In Figure F10 it is clear that addition of the aluminum does not change the laminar burn rate significantly and the aluminum plays a minor role in deflagration rate of the HPP and PS-4. This is not surprising as the aluminum is expected to react in the gas phase, after the initial deflagration wave passes by the material. However, the aluminum will significantly raise the local temperature which will result in a larger pressure change during the course of the burn. In fact, HPP and PS-4 show a pressure rise of one strand that is nearly twice that observed in a strand of Tal-1503.

There are two notable observations concerning the nature of the TAL-1503 burns (1) there is no deconsolidative burning at elevated pressures and (2) there is no dramatic slope change between the low and high pressure regimes. Deconsolidative burning is a mechanism observed at elevated pressures (i.e. P $>150 \mathrm{MPa}$ ) for some materials in which the material begins to deconsolidate during the burn resulting in cracks and fissures in the remaining, unburnt material. The presence of these cracks and fissures allows for convective heat and flame spread and results in a burn that is orders-of-magnitude faster than the laminar burn front. Studies indicate that materials that experience deconsolidative burning are also more violent in thermal explosion tests. ${ }^{3}$ The smooth laminar nature of the Tal-1503 burns indicate that the material burns in a laminar nature up to ca. $300 \mathrm{MPa}$.

In both HPP and PS-4 there is a change of slope in the burn as a function of pressure. Figure F10 shows clearly the slope change for both of the AP/Al propellants. HPP transitions from a small burn exponent to a larger burn exponent as a function of pressure. The PS-4 has an unusual hysteresis in its burn behavior: burns that begin at lower pressures follow one burn law and burns that begin at higher pressure follow a different burn law. Curiously, the lower pressure burn will follow this burn law even if it moves into the high pressure regime; thus it is the initial pressure that dictates the burn law for PS-4. In contrast, TAL-1503 does not appear to have a slope break over the pressure regime studied. Further work, however, is necessary to establish if and where the material burn changes slope. 


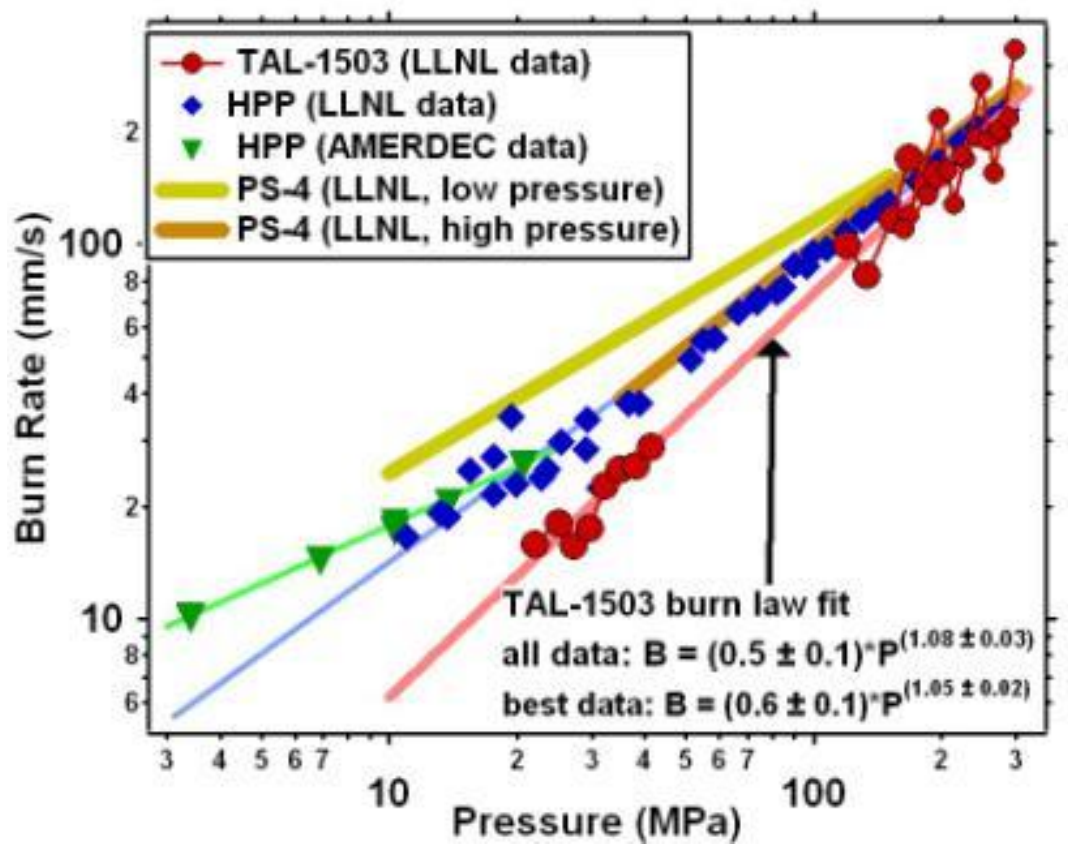

Figure F10. Results of TAL-1503 (an AP/binder formulation) pressure dependent deflagration rate measurements (in red). Also included are results for HPP (an AP/Al/binder formulation) and PS-4 (an AP/Al/binder formulation).

\section{Conclusion}

High pressure deflagration rate measurements of a unique AP/HTPB based material (TAL-1503) were performed using the LLNL high pressure strand burner apparatus. The material burns in a well behaved, laminar fashion between 20 and $300 \mathrm{MPa}$ with a burn law of

$$
B=(0.6 \pm 0.1) \times P^{(1.05 \pm 0.02)}
$$

that was calculated based on the best data available from the experiments. In these results, $B$ is the burn rate in mm/s and $P$ is the pressure in units of MPa. Comparison of the TAL-1503 results with similar propellants that contain micrometer sized aluminum indicate that the burn rates are relatively unaffected by the aluminum. However, the pressure change is significantly larger when aluminum is present, most likely due to the high temperatures achieved from burning aluminum.

\section{Acknowledgements}

We gratefully acknowledge Heidi Turner, Gary Hust, Peter Nunes, and Peter Hsu for their contributions to the small scale safety testing and Nammo Talley (specifically Mark Harris) for supplying the TAL-1503 parts. This work performed under the auspices of the U.S. Department of Energy by Lawrence Livermore National Laboratory under Contract DE-AC52-07NA27344.

\section{References}

1. J. L. Maienschein and J. B. Chandler."High Pressure Laminar Burn Rates of AP/Al/HTPB Propellants - Results from PS-Series" JANNAF 36th Combustion subcommittee Air breathing Propulsion and Propulsion Systems Hazards Subcommittee, Cocoa Beach, Fl, 1999,1.

2. J. L. Maienschein, J. F. Wardell, M. R. DeHaven and C. K. Black, "Deflagration of HMX-Based Explosives at High Temperatures and Pressures" Propellants, Explosives, Pyrotechnics, 2004, 29, 287.

3. J. L. Maienschein, J. F. Wardell, R. K. Weese, B. J. Cuningham and T. D. Tran."Understanding and Predicting the Thermal Explosion Violence of HMX-based and RDX-based Explosives- Experimental Measurements of Material Properties and Reaction Violence" 12 Int. Det. Symp., San Diego, CA, Office of Naval Research, $2002,846$. 